\title{
Impact of Improper Gaussian Signaling on Hardware Impaired Systems
}

\author{
Sidrah Javed ${ }^{1}$, Osama Amin ${ }^{1}$, Salama S. Ikki ${ }^{2}$ and Mohamed-Slim Alouini ${ }^{1}$ \\ ${ }^{1}$ King Abdullah University of Science and Technology (KAUST), Thuwal, Makkah Province, Saudi Arabia. \\ E-mail: \{sidrah.javed, osama.amin, slim.alouini\}@kaust.edu.sa \\ ${ }^{2}$ Department of Electrical Engineering, Faculty of Engineering, Lakehead University, Ontario, Canada. \\ E-mail: sikki@lakeheadu.ca
}

\begin{abstract}
In this paper, we accurately model the hardware impairments (HWI) as improper Gaussian signaling (IGS) which can characterize the asymmetric characteristics of different HWI sources. The proposed model encourages us to adopt IGS scheme for transmitted signal that represents a general study compared with the conventional scheme, proper Gaussian signaling (PGS). First, we express the achievable rate of HWI systems when both PGS and IGS schemes are used when the aggregate effect of HWI is modeled as IGS. Moreover, we tune the IGS statistical characteristics to maximize the achievable rate. Then, we analyze the outage probability for both schemes and derive closed form expressions. Finally, we validate the analytic expressions through numerical and simulation results. In addition, we quantify through the numerical results the performance degradation in the absence of ideal transceivers and the gain reaped from adopting IGS scheme compared with PGS scheme.
\end{abstract}

\section{INTRODUCTION}

Exponentially growing demand for mobile data traffic with improved quality-of-service is one of the main features of the next generation wireless communications. Immense research has been carried out to develop new techniques/configurations in order to support the expected rapidly growing demand for high data rates [1]. However, the performance of the proposed systems can be affected by the non-ideal operation of radio frequency transceivers. Hardware impairments can distort the desired signal, which leads to a major limitation to the overal system performance measured by the acheivable rate or outage probability [2].

Hardware impairments (HWI) arise in different radio frequency and baseband stages such as imperfections in high power amplifier, low noise amplifier non-linearity, inphase and quadrature $(\mathrm{I} / \mathrm{Q})$ imbalance in the radio frequency front-end and phase noise, etc [2], [3]. Therefore, different compenstation schemes and algorithms are developed to dampen the aforementioned imperfections for different configurations [4][6]. In [4], Boulogeorgos et al. proposed I/Q-imbalance selfinterference coordination scheme to mitigate the imbalance effect and improve the diversity order but at a cost of reduced transmission rate. Additionally, In [5], Awadin et al. adopted an opportunistic relaying approach to deal with I/Q-imbalance and other interferences. Moreover in [6], Xia et al. considered massive multiple-input multiple-ouput antenna array system and proposed hardware impairments aware transceiver scheme, to mitigate the distortion noises, by exploiting the statistical knowledge of the channels and the participating antenna arrays [6]. Evaluating different compensation schemes and analyzing the system performance under HWI requires statistical modeling of these imperfections.

The accumulative effect of all forms of hardware imperfections is modelled as a zero mean circularly-symmetric complex Gaussian random variables [6]-[9]. However. this modeling does not capture the asymmetric characteristics of different HWI such as I/Q imbalance. The impaired signal due to I/Q imbalance follows a widely linear transformation of the original signal [4]. Therefore, modeling the aggregate effect of HWI as a zero mean circularly-symmetric complex Gaussian random variables is not accurate because this model does not capture the asymmetric characteristics of the HWI. As such, we propose modeling the aggregate effect of HWI as an improper Gaussian signal (IGS) to allow the asymmetric characteristics of the HWI.

IGS is a generalized complex Gaussian scehme that is appropriately described by the variance as well as the pseudovariance in contrast to its counterpart traditional Gaussian scheme, known as proper Gaussian signaling (PGS) sceheme, which is only described by its variance [10]. Hence, IGS offers additional degrees of design freedom pertinent to its circularly asymmetric characteristics. IGS scheme has already been proven to evidently improve system performance in various system configurations such as cognitive radio systems [11][14], full duplex relaying [15] and alternating relaying [16].

In this paper, we propose modeling the HWI as IGS, then we analyze the achievable rate under the accurate model. Moreover, we adopt asymmetric transmission signals to equalize the effect of HWI through maximizing the achievable rate performance. In addition, we derive closed form expression of the outage probability performance for HWI systems when transmitted signals are chosen from both PGS and IGS. Finally, we validate the derived analysis of the system performance using numerical results.

\section{SySTEM DESCRIPTION}

Consider a conventional single-input single-output system where a transmitter intends to communicate with a receiver through a wireless channel as shown in Fig. 1. The aggregate effect of hardware impairments is considered at both transmitter and receiver. In this section, we first introduce the adopted statistical signal characteristics, then, we present the mathematical model of hardware impairments. 


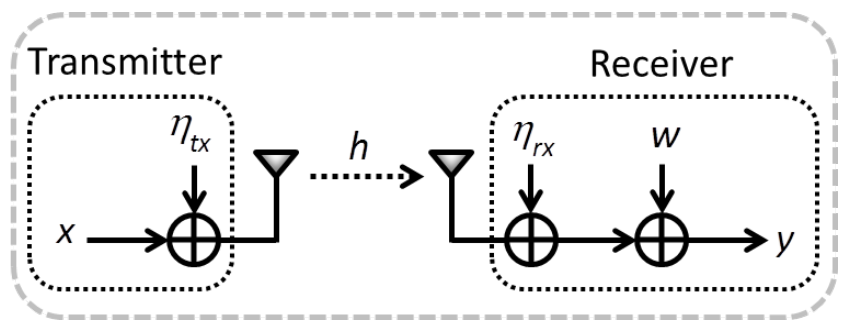

Fig. 1. Hardware Impaired SISO System Model.

\section{A. Statistical Signal Model}

To characterize the difference between PGS and IGS, we consider a complex Gaussian random variable $x$ and introduce the following definitions:

Definition 1. The variance and the pseudo-variance of $x$ are defined, respectively, as $\sigma_{x}^{2}=\mathbb{E}\left[|x|^{2}\right]$ and $\tilde{\sigma}_{x}^{2}=\mathbb{E}\left[x^{2}\right]$, where $\mathbb{E}[$.$] is the expected value operator [17].$

Definition 2. A complex random variable is called proper if its pseudo-variance is equal to zero, otherwise it is called improper [17].

Definition 3. Circularity coefficient $\mathcal{C}_{x}$ of a random variable $x$ is defined as the ratio of the absolute pseudo-variance of $x$ and its variance, i.e., $\mathcal{C}_{x}=\left|\tilde{\sigma}_{x}^{2}\right| / \sigma_{x}^{2}$, where $0 \leq \mathcal{C}_{x} \leq 1$ [18].

The circularity coefficient measures the degree of impropriety or symmetry of $x, \mathcal{C}_{x}=0$ indicates proper signal or symmetric and $\mathcal{C}_{x}=1$ indicates maximally improper or maximally asymmetric signal.

Definition 4. The complex Gaussian random variable $x$ can be fully described as, $x \sim C N\left(m_{x}, \sigma_{x}^{2}, \tilde{\sigma}_{x}^{2}\right)$, where $m_{x}$ is the mean of $x$.

\section{B. Distortion Model}

In this subsection, we describe the mathematical model of the accumulated radio frequency and baseband HWI for a single link. First, we consider the ideal transceivers conventional scenario with no HWI, where a complex symmetric information signal $x_{\mathrm{ID}}$ is transmitted with $x_{\mathrm{ID}} \sim C N\left(0, \sigma_{x}^{2}, 0\right)$. The received signal is expressed as

$$
y_{\mathrm{ID}}=\sqrt{p} h x_{\mathrm{ID}}+w,
$$

where $p$ is the transmitted power, $h \sim C N(0, \pi, 0)$ is the fading channel, and $w$ is the additive white complex Gaussian noise (AWGN) with $w \sim C N\left(0, \sigma_{w}^{2}, 0\right)$.

Practically, radio frequency transceivers introduce hardware impairments which cannot be modeled merely as an additive noise because of their distinct behavior. The aggregate effect of these hardware impairments at the transmitter and the receiver over a flat fading channel appears in the received signal as follows [2].

$$
y=\sqrt{p} h\left(x+\eta_{\mathrm{tx}}\right)+\eta_{\mathrm{rx}}+w
$$

where $x$ is the complex asymmetric transmitted signal with $x \sim C N\left(0, \sigma_{x}^{2}, \tilde{\sigma}_{x}^{2}\right), \eta_{\mathrm{tx}}$ and $\eta_{\mathrm{rx}}$ are the HWI noise at the transmitter and the receiver, respectively.
Lemma 1. The statistical model of the HWI noise that captures its asymmetric nature and characterizes its statistical properties accurately is IGS.

Proof: Consider the I/Q imbalance as a source of HWI and $x_{0}$ is the transmitted signal with $x_{0} \sim C N\left(0, \sigma_{x}^{2}, 0\right)$. The resulting down-converted I/Q signal is expressed as [4]

$$
x_{\mathrm{IQI}}=\alpha_{1} x_{0}+\alpha_{2} x_{0}^{*},
$$

where $x_{0}^{*}$ is the conjugate of $x_{0}$. The transformation in (3) represents a widely linear transformation of $x_{0}$ [10]. The widely linear transformation of PGS results in IGS which can be verified by finding $\tilde{\sigma}_{x_{\mathrm{IQI}}}^{2}$ that is found from $\tilde{\sigma}_{x_{\mathrm{IQI}}}^{2}=2 \alpha_{1} \alpha_{2} \sigma_{x}^{2}$.

As a result, the HWI noise at the transmitter is modeled as a random variable with $\eta_{\mathrm{tx}} \sim C N\left(0, \kappa_{\mathrm{tx}}, \tilde{\kappa}_{\mathrm{tx}}\right)$ where $\left|\tilde{\kappa}_{\mathrm{tx}}\right| \leq$ $\kappa_{\mathrm{tx}} \leq \sigma_{x}^{2}$. On the other hand, it is modeled at the receiver side as $\eta_{\mathrm{rx}} \sim C N\left(0, p|h|^{2} \kappa_{\mathrm{rx}}, p h^{2} \tilde{\kappa}_{\mathrm{rx}}\right)$, where $\left|\tilde{\kappa}_{\mathrm{rx}}\right| \leq \kappa_{\mathrm{rx}} \leq \sigma_{x}^{2}$.

Lemma 2. The equivalent aggregate effect of transceiver impairments is given based on (2) by the following generalized channel model

$$
y=\sqrt{p} h(x+\eta)+w,
$$

where $\eta \sim C N\left(0, \kappa, \tilde{\sigma}_{\eta}^{2}\right), \kappa=\kappa_{\mathrm{tx}}+\kappa_{\mathrm{rx}}$ and $\tilde{\sigma}_{\eta}^{2}=\tilde{\kappa}_{\mathrm{tx}}+\tilde{\kappa}_{\mathrm{rx}}$

Proof: For a flat fading channel, the aggregate effect of the impairments at the receiver has a power that is computed from (2) as

$$
\mathbb{E}\left[\left|\sqrt{p} h \eta_{\mathrm{tx}}+\eta_{\mathrm{rx}}\right|^{2}\right]=p|h|^{2}\left(\kappa_{\mathrm{tx}}+\kappa_{\mathrm{rx}}\right) .
$$

As for the pseudo variance of the aggregate HWI, it is expressed as

$$
\mathbb{E}\left[\left(\sqrt{p} h \eta_{\mathrm{tx}}+\eta_{\mathrm{rx}}\right)^{2}\right]=p h^{2}\left(\tilde{\kappa}_{\mathrm{tx}}+\tilde{\kappa}_{\mathrm{rx}}\right) .
$$

Eventually, the variance of the aggregate asymmetric distortions, $\eta$, is found to be from (5) as $\kappa=\kappa_{\mathrm{tx}}+\kappa_{\mathrm{rx}}$. As for pseudo variance of $\eta$, it is expressed from (6) as $\tilde{\sigma}_{\eta}^{2}=\tilde{\kappa}_{\mathrm{tx}}+\tilde{\kappa}_{\mathrm{rx}}$. Therefore, the signal model in (2) can be equivalently modelled as in (4).

It is important to note that (4) reduces to (1) in case of ideal hardware i.e. $\kappa=0$, which is induced by imposing $\kappa_{\mathrm{tx}}=$ $\kappa_{\mathrm{rx}}=0$ and also $\tilde{\sigma}_{\eta}^{2}=0$, which is deduced from Definition 3.

\section{Achievable Rate}

Based on the adopted signal model in (4), the received signal $y$ consists of the desired signal $\sqrt{p} h x$ and an undesired signal, which is a combination of distortion signal and the thermal noise at the receiver. The undesired signal component is given by $z$ in (7)

$$
z=\sqrt{p} h \eta+w
$$

The end-to-end achievable rate of the presented system is given by $[10]$,

$$
R=\frac{1}{2} \log _{2} \frac{\sigma_{y}^{4}-\left|\tilde{\sigma}_{y}^{2}\right|^{2}}{\sigma_{z}^{4}-\left|\tilde{\sigma}_{z}^{2}\right|^{2}},
$$

where $\tilde{\sigma}_{y}^{2}$ and $\tilde{\sigma}_{z}^{2}$ represent the pseudo-variance of the received and the undesired signal, respectively. In the rest of this 
section, we first express the achievable rate expression in case of ideal hardware, then we present the practical scenario of non-ideal hardware. The achievable rate expressions of the non-ideal system model are developed assuming transmitted signal to be PGS and IGS.

\section{A. Ideal Hardware}

In the absence of HWI, the only undesired signal is the thermal noise present at the receiver, thus, the achievable rate expression reduces to the well known expression in case of PGS in AWGN channel as

$$
R\left(\sigma_{x}^{2}\right)=\log _{2}\left(1+\frac{p|h|^{2} \sigma_{x}^{2}}{\sigma_{w}^{2}}\right)
$$

\section{B. Non-Ideal Hardware with Proper Gaussian Signaling}

Implementing ideal hardware is practically impossible. Moreover, there is always some residual HWI after adopting imperfection mitigating schemes. Hence the aggregate effect of these hardware impairments and thermal noise forms undesired signals as can be seen in (7). Considering the asymmetric nature of this undesired signal component as discussed in Lemmas 1 and 2, the achievable rate when PGS is adopted reduces to

$$
R_{\mathrm{PGS}}\left(\sigma_{x}^{2}\right)=\frac{1}{2} \log _{2} \frac{\left(p|h|^{2}\left(\sigma_{x}^{2}+\kappa\right)+\sigma_{w}^{2}\right)^{2}-\left|p h^{2} \tilde{\sigma}_{\eta}^{2}\right|^{2}}{\left(p|h|^{2} \kappa+\sigma_{w}^{2}\right)^{2}-\left|p h^{2} \tilde{\sigma}_{\eta}^{2}\right|^{2}} .
$$

\section{Non-Ideal Hardware with Improper Gaussian Signaling}

Adopting the IGS provides an additional design parameter i.e. the pseudo-variance of the transmit signal $\left(\tilde{\sigma}_{x}^{2}\right)$. Hence, taking the asymmetric nature of the desired signal component, the achievable rate expression in (8) reduces to

$$
\begin{aligned}
& R_{\mathrm{IGS}}\left(\sigma_{x}^{2}, \tilde{\sigma}_{x}^{2}\right)= \\
& \frac{1}{2} \log _{2} \frac{\left(p|h|^{2}\left(\sigma_{x}^{2}+\kappa\right)+\sigma_{w}^{2}\right)^{2}-\left|p h^{2}\left(\tilde{\sigma}_{x}^{2}+\tilde{\sigma}_{\eta}^{2}\right)\right|^{2}}{\left(p|h|^{2} \kappa+\sigma_{w}^{2}\right)^{2}-\left|p h^{2} \tilde{\sigma}_{\eta}^{2}\right|^{2}} .
\end{aligned}
$$

The achievable rate expression for non-ideal hardware with IGS in (11) can be improved by optimizing $\tilde{\sigma}_{x}^{2}$ as follows

$$
\begin{aligned}
& \text { P1: } \max _{\tilde{\sigma}_{\mathrm{x}}^{2}} R\left(\sigma_{x}^{2}, \tilde{\sigma}_{x}^{2}\right) \\
& \text { s. t. } \quad 0 \leq\left|\tilde{\sigma}_{x}^{2}\right| \leq \sigma_{x}^{2} .
\end{aligned}
$$

The objective function in problem P1 is a concave function in $\tilde{\sigma}_{x}^{2}$, as illustrated in Appendix A. Moreover, the constraint in $\mathbf{P 1}$ is adopted based on the Definition 3 in section II-A.

Theorem 1. The achievable rate of HWI systems is maximized, when adopting IGS scheme, by tuning the transmitted signal characteristics such that $\tilde{\sigma}_{x}^{2}=-\tilde{\sigma}_{\eta}^{2}$.

Proof: The proof is given in Appendix A.
Therefore, the maximum achievable rate of the IGS-based scheme is then given by

$$
R_{\mathrm{IGS}}^{*}\left(\sigma_{x}^{2}\right)=\frac{1}{2} \log _{2} \frac{\left(p|h|^{2}\left(\sigma_{x}^{2}+\kappa\right)+\sigma_{w}^{2}\right)^{2}}{\left(p|h|^{2} \kappa+\sigma_{w}^{2}\right)^{2}-\left|p h^{2} \tilde{\sigma}_{\eta}^{2}\right|^{2}} .
$$

\section{Outage Probability Analysis}

In this section, we analyze the outage performance of the proposed system for the three different scenarios, ideal hardware, non-ideal hardware with PGS and non-ideal hardware with IGS. The system outage is declared when the instantaneous rate falls below a certain threshold target rate $\mathrm{R}_{\mathrm{th}}$. Therefore, the outage probability $P_{\text {out }}$ is defined as

$$
P_{\text {out }}\left(R_{\mathrm{th}}\right)=\operatorname{Pr}\left\{R<R_{\mathrm{th}}\right\},
$$

where $\operatorname{Pr}\{A\}$ defines the probability of occurrence of event A.

It is worth to emphasize that although studying the SNRbased outage analysis is equivalent to the rate-based outage for the ideal hardware scenario, it is not the same for nonideal hardware scenarios. Therefore, considering rate-based outage is imperative to have a fair comparison study of different scenarios.

\section{A. Ideal Hardware}

The ideal hardware scenario provides a lower bound on the outage probability performance of systems with HWI. To this end, the outage probability of Raleigh fading channel with a target rate $R_{\mathrm{th}}$ bits $/ \mathrm{sec} / \mathrm{Hz}$ is given as

$$
\begin{aligned}
P_{\text {out }}\left(R_{\mathrm{th}}\right) & =\operatorname{Pr}\left\{\gamma \leq 2^{R_{\mathrm{th}}}-1\right\} \\
& =1-\exp \left(-\frac{\sigma_{w}^{2}\left(2^{R_{\mathrm{th}}}-1\right)}{\pi p \sigma_{x}^{2}}\right)
\end{aligned}
$$

where $\gamma=p|h|^{2} \sigma_{x}^{2} / \sigma_{w}^{2}$, which has an exponential distribution with mean $p \pi \sigma_{x}^{2} / \sigma_{w}^{2}$. Thus, the outage probability reduces to

$$
P_{\text {out }}\left(R_{\mathrm{th}}\right)=1-\exp \left(-\frac{\sigma_{w}^{2}\left(2^{R_{\mathrm{th}}}-1\right)}{\pi p \sigma_{x}^{2}}\right) \text {. }
$$

\section{B. Non-Ideal Hardware with Proper Gaussian Signaling}

In case of non-ideal hardware with symmetric signaling, the outage probability $P_{\text {out,p }}$ is given by the following theorem.

Theorem 2. In single link systems that adopts PGS under $H W I$, the outage probability of the Rayleigh fading channel with a target rate $R_{\mathrm{th}} \mathrm{bits} / \mathrm{sec} / \mathrm{Hz}$ is given by

$$
P_{\text {out }, \mathrm{p}}\left(R_{\mathrm{th}}\right)= \begin{cases}1-e^{-\frac{\sigma_{w}^{2}}{\pi} \gamma_{1}} & \text { if } 0 \leq R_{\mathrm{th}} \leq R_{1} \\ 1 & \text { if } R_{1} \leq R_{\mathrm{th}}<\infty\end{cases}
$$

where $\gamma_{1}$ is expressed as

$\gamma_{1}=\frac{-\sigma_{x}^{2}-\left(1-2^{2 R_{\mathrm{th}}}\right) \kappa+\sqrt{2^{2 R_{\mathrm{th}}} \sigma_{x}^{4}+\left(1-2^{2 R_{\mathrm{th}}}\right)^{2}\left|\tilde{\sigma}_{\eta}^{2}\right|^{2}}}{p\left[\left(1-2^{2 R_{\mathrm{th}}}\right)\left(\kappa^{2}-\left|\tilde{\sigma}_{\eta}^{2}\right|^{2}\right)+2 \kappa \sigma_{x}^{2}+\sigma_{x}^{4}\right]}$ 
and $R_{1}$ is found from

$$
R_{1}=\frac{1}{2} \log _{2}\left(\frac{\left(\sigma_{x}^{2}+\kappa\right)^{2}-\left|\tilde{\sigma}_{\eta}^{2}\right|^{2}}{\kappa^{2}-\left|\tilde{\sigma}_{\eta}^{2}\right|^{2}}\right)
$$

Proof: By substituting (10) in (13), yields outage probability as given by (19).

$$
P_{\text {out }, \mathrm{p}}\left(R_{\mathrm{th}}\right)=\operatorname{Pr}\left\{a_{\mathrm{p}} \gamma^{2}+b \gamma+c \leq 0\right\}
$$

where, $a_{\mathrm{p}}=p^{2}\left(\left(1-2^{2 R_{\mathrm{th}}}\right)\left(\kappa^{2}-\left|\tilde{\sigma}_{\eta}^{2}\right|^{2}\right)+2 \kappa \sigma_{x}^{2}+\sigma_{x}^{4}\right)$, $b=2 p\left(\left(1-2^{2 R_{\mathrm{th}}}\right) \kappa+\sigma_{x}^{2}\right), c=\left(1-2^{2 R_{\mathrm{th}}}\right)$ and $\gamma=p|h|^{2} / \sigma_{w}^{2}$.

Let $f_{\mathrm{PGS}}(\gamma)=a_{\mathrm{p}} \gamma^{2}+b \gamma+c$. To solve (19), we need to investigate the cases at which $f_{\mathrm{PGS}}(\gamma) \leq 0$. First, having $R_{\text {th }}>0$ implies $c \leq 0$, thus different possible cases for $f_{\mathrm{PGS}}(\gamma) \leq 0$ is determined based on $a_{\mathrm{p}}$ and $b$ as follows

1) Convex Quadratic $\left(a_{\mathrm{p}} \geq 0\right)$ : In this case, $f_{\mathrm{PGS}}(\gamma)$ represents convex function in $\gamma$ and the equality $f_{\mathrm{PGS}}(\gamma)=0$ possesses only one non-negative zero $\gamma_{1}$, that is given by (17), irrespective of the value of b. Therefore, we can find the outage probability by finding the probability of event when $0 \leq \gamma \leq \gamma_{1}$, which is given as follows

$$
P_{\text {out }, \mathrm{p}}\left(R_{\mathrm{th}}\right)=1-e^{-\frac{\sigma_{w}^{2}}{\pi} \gamma_{1}} .
$$

The aforementioned expression is valid for $a_{\mathrm{p}} \geq 0$, which reduces to $R_{\mathrm{th}} \leq R_{1}$, where $R_{1}$ is given by (18) after some mathematical manipulations.

2) Concave Quadratic $\left(a_{\mathrm{p}} \leq 0, b \leq 0\right)$ : In this case, $f_{\mathrm{PGS}}(\gamma)<0$ is always valid, which reduces to outage probability equal to 1 . Moreover, the condition $\left(a_{\mathrm{p}} \leq 0, b \leq 0\right.$ and $\left.c \leq 0\right)$ implies that $R_{\mathrm{th}}$ should be greater than $R_{1}$ to incur complete system outage.

3) Concave Quadratic $\left(a_{\mathrm{p}} \leq 0, b \geq 0\right)$ : In this case, $a_{\mathrm{p}} \leq 0$ reduces to $R_{\mathrm{th}} \geq R_{1}$, while $b \geq 0$ reduces to $R_{\mathrm{th}} \leq$ $R_{2}$, which is expressed as

$$
R_{2}=\frac{1}{2} \log _{2}\left(1+\frac{\sigma_{x}^{2}}{\kappa}\right) .
$$

Appendix $\mathrm{B}$ demonstrates $R_{2}<R_{1}$, that results in contradicting conditions. Consequently, this case does not contribute in the overall outage probability expression.

Encompassing all the possible cases of outage occurrence with PGS scheme, which is summarized in Table I, completes the proof of Theorem 2 .

\section{Non-Ideal Hardware with Improper Gaussian Signaling}

In case of non-ideal hardware system that uses IGS scheme, the system outage probability $P_{\text {out,i }}$ is evaluated according to optimized IGS in Theorem 1

$$
P_{\text {out }, \mathrm{i}}\left(R_{\mathrm{th}}\right)=\operatorname{Pr}\left\{R_{\mathrm{IGS}}^{*}<R_{\mathrm{th}}\right\} \text {, }
$$

which is given by the following theorem.
TABLE I. CLOSED FORM EXPRESSIONS OF THE OUTAGE PROBABILITY WITH PGS AND IGS SCHEMES FOR VARIOUS TARGET RATE REGIONS

\begin{tabular}{cccc}
\hline \hline Signaling & Cases & Target rate region & $P_{\text {out }}$ \\
\hline \multirow{4}{*}{ PGS } & Convex $\left(a_{\mathrm{p}} \geq 0\right)$ & $0 \leq R_{\mathrm{th}} \leq R_{1}$ & $1-e^{-\frac{\sigma_{w}^{2}}{\pi} \gamma_{1}}$ \\
& Concave $\left(a_{\mathrm{p}} \leq 0, \mathrm{~b} \leq 0\right)$ & $R_{1} \leq R_{\mathrm{th}}<\infty$ & 1 \\
& Concave $\left(a_{\mathrm{p}} \leq 0, \mathrm{~b} \geq 0\right)$ & $R_{\mathrm{th}} \leq R_{2} \& R_{\mathrm{th}} \geq R_{1}$ & Infeasible \\
\hline \multirow{4}{*}{ IGS } & Convex $\left(a_{\mathrm{I}} \geq 0\right)$ & $0 \leq R_{\mathrm{th}} \leq R_{3}$ & $1-e^{-\frac{\sigma_{w}^{2}}{\pi} \gamma_{2}}$ \\
& Concave $\left(a_{\mathrm{I}} \leq 0, \mathrm{~b} \leq 0\right)$ & $R_{3} \leq R_{\mathrm{th}}<\infty$ & 1 \\
& Concave $\left(a_{\mathrm{I}} \leq 0, \mathrm{~b} \geq 0\right)$ & $R_{\mathrm{th}} \leq R_{2} \& R_{\mathrm{th}} \geq R_{3}$ & Infeasible \\
\hline \hline
\end{tabular}

Theorem 3. The outage probability of a single link Rayleigh fading communication system that uses IGS to achieve a target rate $R_{\mathrm{th}} \mathrm{bits} / \mathrm{sec} / \mathrm{Hz}$ under $H W I$ is given by

$$
P_{\text {out }, \mathrm{i}}\left(R_{\mathrm{th}}\right)=\left\{\begin{array}{lr}
1-e^{-\frac{\sigma_{w}^{2}}{\pi} \gamma_{2}} & \text { if } 0 \leq R_{\mathrm{th}} \leq R_{3} \\
1 & \text { if } R_{3} \leq R_{\mathrm{th}}<\infty
\end{array}\right.
$$

where,

$$
\gamma_{2}=\frac{-\sigma_{x}^{2}-\left(1-2^{2 R_{\mathrm{th}}}\right) \kappa+2^{R_{\mathrm{th}}} \sqrt{\sigma_{x}^{4}-\left(1-2^{2 R_{\mathrm{th}}}\right)\left|\tilde{\sigma}_{\eta}^{2}\right|^{2}}}{p\left[\left(\sigma_{x}^{2}+\kappa\right)^{2}-2^{2 R_{\mathrm{th}}}\left(\kappa^{2}-\left|\tilde{\sigma}_{\eta}^{2}\right|^{2}\right)\right]}
$$

and

$$
R_{3}=\frac{1}{2} \log _{2}\left(\frac{\left(\sigma_{x}^{2}+\kappa\right)^{2}}{\kappa^{2}-\left|\tilde{\sigma}_{\eta}^{2}\right|^{2}}\right)
$$

Proof: By substituting the optimized rate expression $R_{\mathrm{IGS}}^{*}$ from (12) in (22), yields outage probability as given by (26).

$$
P_{\text {out }, \mathrm{i}}\left(R_{\mathrm{th}}\right)=\operatorname{Pr}\left\{a_{\mathrm{I}} \gamma^{2}+b \gamma+c \leq 0\right\}
$$

where, $a_{\mathrm{I}}=p^{2}\left(\left(1-2^{2 R_{\mathrm{th}}}\right) \kappa^{2}+2^{2 R_{\mathrm{th}}}\left|\tilde{\sigma}_{\eta}^{2}\right|^{2}+2 \kappa \sigma_{x}^{2}+\sigma_{x}^{4}\right)$ and $\gamma=p|h|^{2} / \sigma_{w}^{2}$.

Let $f_{\text {IGS }}(\gamma)=a_{\mathrm{I}} \gamma^{2}+b \gamma+c$. To solve (26), detailed investigation has been carried out to enumerate all cases at which $f_{\text {IGS }}(\gamma) \leq 0$. It is important to highlight that the coefficients $b$ and $c$ remain the same for both PGS and IGS schemes. Given, $R_{\mathrm{th}}>0$ implies $c \leq 0$, thus different possible cases for $f_{\text {IGS }}(\gamma) \leq 0$ are analyzed based on $a_{\text {I }}$ and $b$ as follows

1) Convex Quadratic $\left(a_{\mathrm{I}} \geq 0\right)$ : In this case, $f_{\mathrm{IGS}}(\gamma)$ is evidently a convex function in $\gamma$. Regardless of the value of $\mathrm{b}$, the equality $f_{\mathrm{IGS}}(\gamma)=0$ possesses only one non-negative zero $\gamma_{2}$, that is given by (24). Consequently, we can find the outage probability $P_{\text {out,i }}$ by finding the probability of event when $0 \leq \gamma \leq \gamma_{2}$, which is given in closed-form expression as

$$
P_{\text {out }, \mathrm{i}}\left(R_{\mathrm{th}}\right)=1-e^{-\frac{\sigma_{w}^{2}}{\pi} \gamma_{2}}
$$

The above stated expression is valid for $a_{\mathrm{I}} \geq 0$, which reduces to $R_{\mathrm{th}} \leq R_{3} . R_{3}$ stated by (25), is a function of signal power and HWI characteristics.

2) Concave Quadratic $\left(a_{\mathrm{I}} \leq 0, b \leq 0\right)$ : In this case, $f_{\text {IGS }}(\gamma)<0$ is always valid $\forall \gamma \geq 0$, which imparts complete system outage i.e. $P_{\text {out }, \mathrm{i}}=1$. Furthermore, 


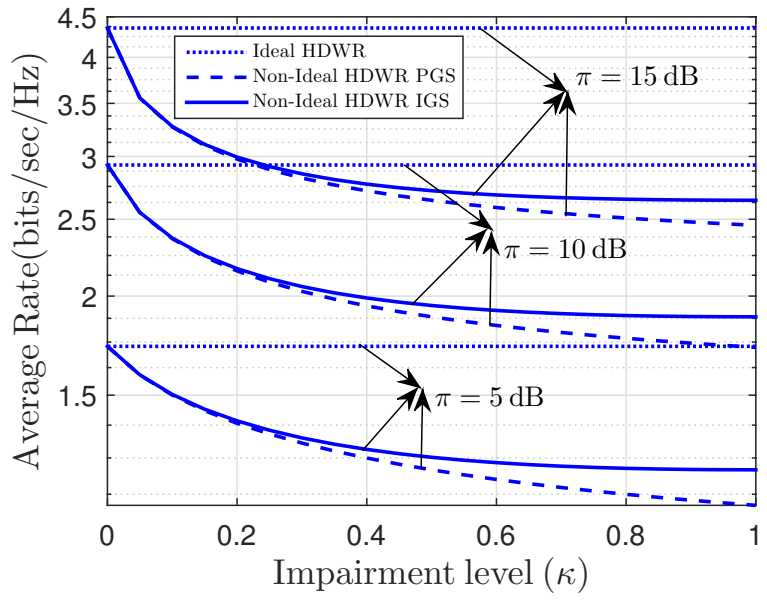

Fig. 2. Average achievable rate vs. Impairment levels for various CSI

the condition $\left(a_{\mathrm{I}} \leq 0, b \leq 0\right.$ and $\left.c \leq 0\right)$ implies that $R_{\text {th }}$ should be greater than $R_{3}$ to result in overall system outage.

3) Concave Quadratic $\left(a_{\mathrm{I}} \leq 0, b \geq 0\right)$ : This case implies two constraints on the target achievable rate. Firstly, $a_{\mathrm{I}} \leq 0$ reduces to $R_{\mathrm{th}} \geq R_{3}$, whereas $b \geq 0$ reduces to $\bar{R}_{\mathrm{th}} \leq R_{2}$, where $R_{2}$ is given by (20).

Following the similar steps as in Appendix B, one can prove that $R_{2}<R_{3}$. This results in contradicting conditions. Therefore, this case does not participate in the overall outage probability expression for $P_{\text {out }, \mathrm{i}}$.

Encompassing all the possible cases of outage occurrence with IGS scheme, completes the proof of Theorem 3.

The analysis depicts that there are different cutoff rates, for declaring complete outage, $R_{1}$ and $R_{3}$ in case of PGS and IGS respectively. All these cases are summarized in Table I.

\section{Simulation AND Numerical Results}

In this section, we investigate the degradation in the system performance caused by HWI using the accurate impairment modeling as IGS compared to the ideal hardware. Furthermore, we examine the benefits reaped by adopting IGS in mitigating the HWI compared to the PGS scheme. As for the system parameters, we assume, unless otherwise specified, $p=1$, $\sigma_{x}^{2}=\sigma_{w}^{2}=1, \kappa=1$ and $\tilde{\sigma}_{\eta}^{2}=0.9$. In the rest of this section, system performance in terms of achievable rate and outage probability is plotted against average channel gain, target rates and IQI levels for the three aforementioned scenarios: ideal and non-ideal hardware with both the PGS and IGS schemes. Throughout the following examples, we include the simulation results in different line formats and the analytical results using markers to show that both are perfectly aligned.

Firstly, the averaged achievable rate is analyzed at various impairment levels $\kappa$, ranging from 0 to 1 , for three different CSI values in Fig. 2. $\kappa=0$ denotes the ideal hardware, while $\kappa=1$ signifies the maximally non-ideal hardware. It is evident from Fig. 2, that the achievable rate significantly decreases with the increase in impairment level $\kappa$, in case of non-ideal hardware. Furthermore, the deteriorating effect

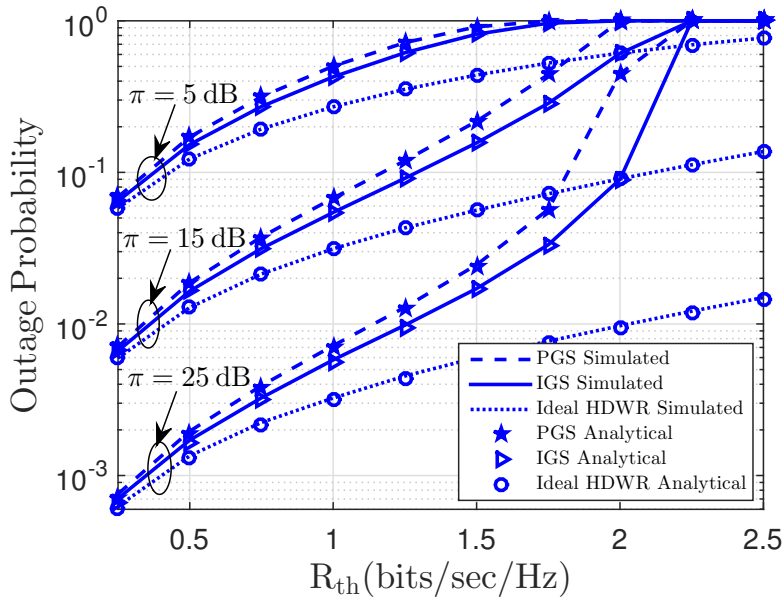

Fig. 3. Outage probability vs. target rates for various CSI

of hardware impairments is considerably mitigated in the presence of IGS as compared to it's counterpart PGS for all CSI values, especially for highly hardware-impaired systems.

Secondly, Fig 3 depicts the result of both simulated and analytical outage probability versus various target rates $\left(R_{\mathrm{th}}\right)$ in case of ideal and non-ideal hardware for various CSI. It is important to note that, analytical expressions are well in accordance with the simulated results for all scenarios. As for the ideal hardware performance, it is evident from figure that it does not declare severe outage till a very large target rate value. On the other hand, hardware impairment degrades the system performance significantly. HWI results in more severe degradation effects at higher target rates and better channel gain. The IGS scheme succeeds to lessen the HWI and improve the system performance compared with the PGS especially at high $\pi$ and $R_{\mathrm{th}}$.

Furthermore, simulated and analytical results of outage probability versus the degree of impairment levels for various target rates are shown in Fig 4. Channel gain is assumed to have a variance of $\pi=10 \mathrm{~dB}$. It is evident that the outage increases with the increase of $\kappa$. Moreover, a rapid increase in the outage probability is observed for higher target rate values while moving towards increasing non-ideal hardware conditions. Eventually, it is worth noting that IGS scheme reaps a huge gain in terms of system outage probability by highly mitigating the effects of HWI especially at higher target rates.

Finally, outage probability in case of ideal and non-ideal hardware versus varying channel states is observed in Fig 5. Outage probability decreases with the increase in channel gain. Evidently, ideal and non-ideal outage probabilities are relatively close to each other for low target rates. Whereas this difference increases for higher target rates and hence some efficient signaling scheme is required to reduce system outage given limited power budget. It is important to highlight that in case of $R_{\mathrm{th}}=2 \mathrm{bits} / \mathrm{sec} / \mathrm{Hz}$, IGS based scheme can operate in medium channel gain to achieve the same performance of PGS based scheme that operates in high channel gain environment. 


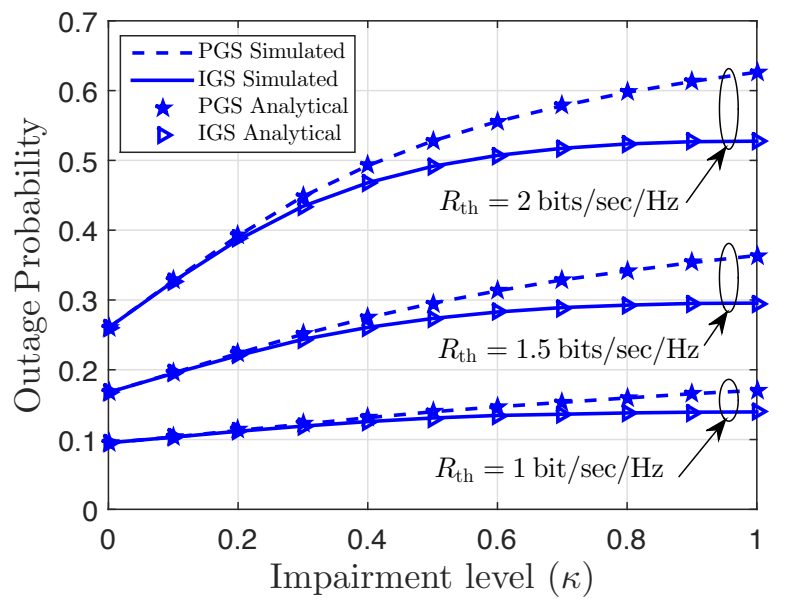

Fig. 4. Outage probability vs. Impairment level for various target rates

\section{CONCLUSION}

In this paper, we proposed a statistical model for communication links with non-ideal hardware. Then, we adopted the IGS scheme transmission in order to reduce the self interference induced by HWI. Closed form expressions for the achievable rate and outage probability are derived in case of ideal and non-ideal hardware with PGS and IGS schemes. Given a specific power budget, IGS scheme provides an additional design parameter, which can be efficiently optimized to improve overall system performance. On the other hand, PGS is merely a function of the transmit power hence it is unable to perform optimally under hardware impairments given limited power budget. Moreover simulation and analytic results depict the gain reaped by IGS scheme referring PGS scheme as a benchmark. Furthermore, numerical results show that the utility of IGS scheme in non-ideal scenario is overwhelming especially for higher impairment levels and higher target rates. Therefore, IGS scheme is a suitable candidate to improve achievable rates and significantly decrease system outage with relatively less transmission power requirement, which makes it appropriate for the future generation of interference limited networks.

\section{APPENDIX A \\ PROOF OF THEOREM 1}

Consider the objective function of the optimization problem $\mathrm{P} 1$, maximizing $R$ is equivalent to maximizing $\psi=2^{2 R}$. Therefore, consider $\psi$, the first derivative is found to be

$$
\frac{\partial \psi}{\partial \tilde{\sigma}_{x}^{2}}=\frac{-2 p^{2}\left|h^{2}\right|^{2}\left(\tilde{\sigma}_{x}^{2}+\tilde{\sigma}_{\eta}^{2}\right)}{\left(p|h|^{2} \kappa+\sigma_{w}^{2}\right)^{2}-\left|p h^{2} \tilde{\sigma}_{\eta}^{2}\right|^{2}} .
$$

Then, by taking second derivative with respect to $\tilde{\sigma}_{x}^{2}$ yields,

$$
\frac{\partial^{2} \psi}{\partial \tilde{\sigma}_{x}^{2}}=\frac{-2 p^{2}\left|h^{2}\right|^{2}}{\left(p|h|^{2} \kappa+\sigma_{w}^{2}\right)^{2}-\left|p h^{2} \tilde{\sigma}_{\eta}^{2}\right|^{2}}<0 .
$$

Therefore, the objective function is strictly concave in $\tilde{\sigma}_{x}^{2}$ since $p>0$. The corresponding optimal solution is obtained by

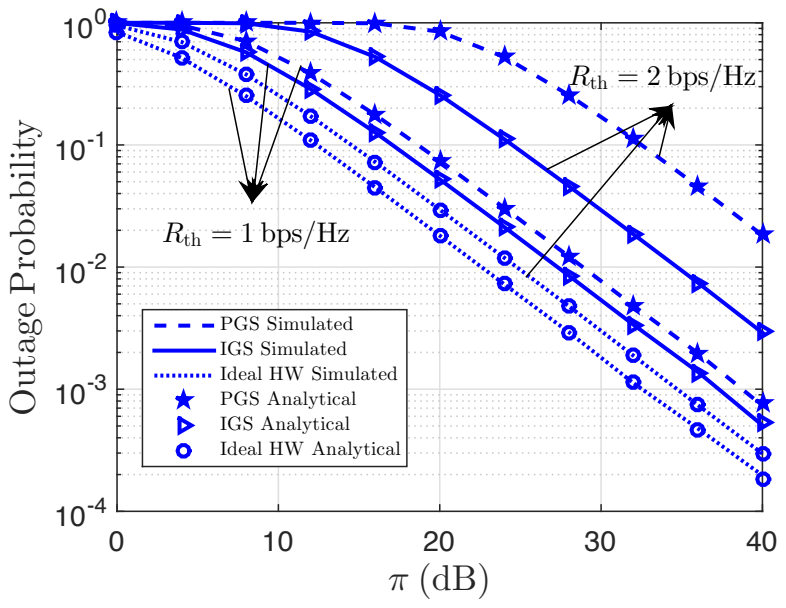

Fig. 5. Outage probability vs. CSI for various target rates

setting $\frac{\partial f}{\partial \tilde{\sigma}^{2}}=0$, as the stationary point is necessary and sufficient $\mathrm{K} K \mathrm{KT}$ condition, for optimality, in the given scenario. As a result, we obtain $\tilde{\sigma}_{x}^{2}=-\tilde{\sigma}_{\eta}^{2}$. Generally, $\left|\tilde{\sigma}_{\eta}^{2}\right| \leq \sigma_{x}^{2}$, hence the constraint in the optimization problem $\mathbf{P 1}$ is always satisfied.

\section{APPENDIX B \\ Proof of $R_{2}<R_{1}$ AND $R_{2}<R_{3}$}

The proof of $R_{2} \leq R_{1}$ is equivalent to the proof of $\psi_{2} \leq$ $\psi_{1}$ where $\psi_{k}=2^{2 R_{k}}$ for $k \in(1,2)$. To prove this, first we will show that $\psi_{1}$ is an increasing function in $\left|\tilde{\sigma}_{\eta}^{2}\right|^{2}$. By taking the first derivative of $\psi_{1}$, we obtain

$$
\frac{d \psi_{1}}{d\left|\tilde{\sigma}_{\eta}^{2}\right|^{2}}=\frac{\sigma_{x}^{2}\left(\sigma_{x}^{2}+2 \kappa\right)}{\left(\kappa^{2}-\left|\tilde{\sigma}_{\eta}^{2}\right|^{2}\right)^{2}} \geq 0 \quad \forall 0 \leq\left|\tilde{\sigma}_{\eta}^{2}\right|^{2} \leq \kappa^{2}
$$

which proves that $\psi_{1}$ is an increasing function in $\left|\tilde{\sigma}_{\eta}^{2}\right|^{2}$ for the given range.

Therefore, using (17) the minimum value of $\psi_{1}$ as a function of $\left|\tilde{\sigma}_{\eta}^{2}\right|^{2}$ occurs at $\left|\tilde{\sigma}_{\eta}^{2}\right|^{2}=0$ and is given by

$$
\tilde{\psi}_{1}=\left(1+\frac{\sigma_{x}^{2}}{\kappa}\right)^{2}=\psi_{2}^{2} .
$$

Moreover, to prove $\psi_{2} \leq \psi_{1}$, it is sufficient to prove that $\psi_{2} \leq \tilde{\psi}_{1}$ as $\psi_{2} \leq \tilde{\psi}_{1} \leq \psi_{1}$. Given $\psi_{2} \geq 1$, (31) proves that $\psi_{2} \leq \tilde{\psi}_{1} \leq \psi_{1}$ which in turn implies that $R_{2} \leq R_{1}$. Given non-zero signal power and finite HWI levels, $R_{2}$ is strictly less than $R_{1}$.

Similarly, it can also be proved that $R_{2}<R_{3}$ by following similar steps.

\section{REFERENCES}

[1] M. Dohler, T. Nakamura, A. Osseiran, J. F. Monserrat, O. Queseth, and P. Marsch, 5G Mobile and Wireless Communications Technology. Cambridge University Press, 2016.

[2] T. Schenk, RF imperfections in high-rate wireless systems: impact and digital compensation. Springer Science \& Business Media, 2008. 
[3] R. Krishnan, On the Impact of Phase Noise in Communication Systems-Performance Analysis and Algorithms. Chalmers University of Technology, Apr. 2015.

[4] A.-A. Boulogeorgos, V. Kapinas, R. Schober, and G. Karagiannidis, "I/Q-imbalance self-interference coordination," IEEE Trans. Wireless Commun., vol. 15, no. 6, pp. 4157-4170, June 2016.

[5] M. Awadin, N. Al-Dhahir, and R. Hamila, "OFDM full-duplex DF relaying under I/Q imbalance and loopback self-interference," IEEE Trans. Veh. Technol., vol. 65, no. 8, pp. 6737-6741, Aug. 2016.

[6] X. Xia, D. Zhang, K. Xu, W. Ma, and Y. Xu, "Hardware impairments aware transceiver for full-duplex massive MIMO relaying," IEEE Trans. Signal Process., vol. 63, no. 24, pp. 6565-6580, Dec. 2015.

[7] J. Li, M. Matthaiou, and T. Svensson, "I/Q imbalance in AF dual-hop relaying: Performance analysis in nakagami-m fading," IEEE Trans. Commun., vol. 62, no. 3, pp. 836-847, Mar. 2014.

[8] M. Mokhtar, A. Gomaa, and N. Al-Dhahir, "OFDM AF relaying under I/Q imbalance: Performance analysis and baseband compensation," IEEE Trans. Commun., vol. 61, no. 4, pp. 1304-1313, Apr. 2013.

[9] T. T. Duy, T. Q. Duong, D. B. da Costa, V. N. Q. Bao, and M. Elkashlan, "Proactive relay selection with joint impact of hardware impairment and co-channel interference," IEEE Trans. Commun., vol. 63, no. 5, pp. 1594-1606, May. 2015.

[10] Y. Zeng, C. M. Yetis, E. Gunawan, Y. L. Guan, and R. Zhang, "Transmit optimization with improper Gaussian signaling for interference channels," IEEE Trans. Signal Process., vol. 61, no. 11, pp. 2899-2913, June 2013.

[11] M. Gaafar, O. Amin, W. Abediseid, and M.-S. Alouini, "Spectrum sharing opportunities of full-duplex systems using improper Gaussian signaling," in in Proc. IEEE Int. Symp. on Personal, Indoor and Mobile Radio Communications (PIMRC), Hong Kong, Sep. 2015.

[12] O. Amin, W. Abediseid, and M.-S. Alouini, "Underlay cognitive radio systems with improper gaussian signaling: Outage performance analysis," IEEE Trans. Wireless Commun., vol. 15, no. 7, July 2016.

[13] M. Gaafar, O. Amin, W. Abediseid, and M.-S. Alouini, "Sharing the licensed spectrum of full-duplex systems using improper Gaussian signaling," in in Proc. IEEE Global Communincation Conf. (GLOBECOM), San Diego, Dec. 2015.

[14] O. Amin, W. Abediseid, and M.-S. Alouini, "Outage performance of cognitive radio systems with improper Gaussian signaling," in Proc. IEEE Int. Symp. on Information Theory (ISIT), Hong Kong, Jun. 2015.

[15] M. Gaafar, M. G. Khafagy, O. Amin, and M.-S. Alouini, "Improper gaussian signaling in full-duplex relay channels with residual selfinterference," in Proc. IEEE Int. Conf. Commun. (ICC), Kuala Lumpure, May 2016.

[16] M. Gaafar, O. Amin, A. Ikhlef, A. Chaaban, and M.-S. Alouini, "On alternate relaying with improper gaussian signaling," vol. 20, no. 8, pp. 1683-1686, Aug. 2016.

[17] F. D. Neeser and J. L. Massey, "Proper complex random processes with applications to information theory," IEEE Trans. Inf. Theory, vol. 39, no. 4, pp. 1293-1302, Jul. 1993.

[18] E. Ollila, "On the circularity of a complex random variable," IEEE Signal Process. Lett., vol. 15, pp. 841-844, 2008. 